Article

\title{
On a Class of Generalized Nonexpansive Mappings
}

\author{
Simeon Reich * and Alexander J. Zaslavski \\ Department of Mathematics, The Technion-Israel Institute of Technology, Haifa 32000, Israel; \\ ajzasl@technion.ac.il \\ * Correspondence: sreich@technion.ac.il
}

Received: 5 June 2020; Accepted: 30 June 2020; Published: 3 July 2020

\begin{abstract}
In our recent work we have introduced and studied a notion of a generalized nonexpansive mapping. In the definition of this notion the norm has been replaced by a general function satisfying certain conditions. For this new class of mappings, we have established the existence of unique fixed points and the convergence of iterates. In the present paper we construct an example of a generalized nonexpansive self-mapping of a bounded, closed and convex set in a Hilbert space, which is not nonexpansive in the classical sense.
\end{abstract}

Keywords: banach space; convex set; fixed point; Hilbert space; nonexpansive mapping

MSC: 47H09; 47H10; 54E50

\section{Introduction}

The by now well-developed fixed point theory of nonexpansive operators has stemmed from Stefan Banach's classical result, which was obtained in [1]. The main goals of this theory include establishing the existence of fixed points of nonexpansive operators and the study of the convergence of their orbits to their fixed points [2-16]. This theory also concerns investigations of feasibility, common fixed point problems and variational inequalities [15-21].

In our recent work we have introduced and studied a notion of a generalized nonexpansive mapping. In the definition of this notion the norm has been replaced by a general function satisfying certain conditions. For this new class of mappings, we have established the existence of unique fixed points and the convergence of iterates.

In the present paper, we construct an example of a generalized nonexpansive self-mapping of a bounded, closed and convex set in a Hilbert space, which is not nonexpansive in the classical sense.

To wit, in [22,23] we examine the following class of nonlinear operators.

Let $(X,\|\cdot\|)$ be a Banach space and let $K$ be a bounded, closed and convex subset of $X$. Let $f: X \rightarrow[0, \infty)$ be a continuous function such that $f(0)=0$, the set $f(K-K)$ is bounded, and such that $f$ enjoys the following three properties:

(i) For every positive number $\epsilon$, there is a positive number $\delta$ such that for every pair of points $x, y \in K$ satisfying $f(x-y) \leq \delta$, we have $\|x-y\| \leq \epsilon$;

(ii) For every positive number $\lambda<1$, there is a positive number $\phi(\lambda)<1$ for which

$$
f(\lambda(x-y)) \leq \phi(\lambda) f(x-y) \text { for every pair of points } x, y \in K
$$

(iii) The function $(x, y) \mapsto f(x-y), x, y \in K$, is uniformly continuous on $K \times K$.

Denote by $\mathcal{A}$ the set of all continuous operators $A: K \rightarrow K$ such that

$$
f(A x-A y) \leq f(x-y) \text { for all } x, y \in K .
$$


For every pair of operators $A, B \in \mathcal{A}$, put

$$
d(A, B):=\sup \{\|A x-B x\|: x \in K\} .
$$

Evidently, $(\mathcal{A}, d)$ is a complete metric space.

In [22], we establish the existence of a set $\mathcal{F}$, which is a countable intersection of open and everywhere dense subsets of $\mathcal{A}$, such that each operator $C \in \mathcal{F}$ has a unique fixed point and all its iterates converge uniformly to this fixed point.

At this juncture, it is worthwhile mentioning that the classical theorem of Francesco De Blasi and Józef Myjak [24] is a special case of this result, where the function $f$ is the norm. Clearly, the operators defined above may be considered generalized nonexpansive mappings with respect to the function $f$. Note that in $[25,26]$ this approach was applied to generalized best approximation problems.

In [23] we improve the results of [22]. To wit, we introduce there a notion of a contractive mapping, show that most mappings in $\mathcal{A}$ (in the sense of Baire category) are contractive, every contractive mapping possesses a unique fixed point and that all its iterates converge to this point uniformly. We emphasize that all these results were obtained for a bounded set $K$.

In [27] we extend one of the main results of [23] to unbounded sets. Moreover, to establish this result, which we present in the next section, it turns out that we do not need property (ii).

\section{A Convergence Result}

Let $(X,\|\cdot\|)$ be a Banach space, and let $K$ be a nonempty and closed subset of $X$. Let $f: X \rightarrow[0, \infty)$ be a continuous function with $f(0)=0$ such that $f$ enjoys the following two properties:

(P1) for every positive number $\epsilon$, there is a positive number $\delta$ such that for every pair of points $x, y \in K$ which satisfies $f(x-y) \leq \delta$, we have $\|x-y\| \leq \epsilon$;

(P2) the function $(x, y) \mapsto f(x-y), x, y \in K$, is uniformly continuous on $K \times K$ and for every point $\xi \in K$, the function $f(x-\xi), x \in D$, is bounded on every bounded subset $D$ of $K$.

Next, assume that $A: K \rightarrow K$ is a continuous mapping, $\psi:[0, \infty) \rightarrow[0,1]$ is a decreasing function satisfying

$$
\psi(t)<1 \text { for all } t>0,
$$

and that

$$
f(A x-A y) \leq \psi(f(x-y)) f(x-y) \text { for all } x, y \in K .
$$

In the literature, such an operator $A$ is said to be contractive [14].

In [27] we have established the following theorem.

Theorem 1. The mapping $A$ has a unique fixed point $x_{A} \in K$ and $A^{i} x \rightarrow x_{A}$ as $i \rightarrow \infty$ for all $x \in K$, uniformly on bounded subsets of $K$.

This theorem is also a generalization of the result of [28], which was obtained for the case where $f(x)=\|x\|$.

\section{An Example}

Let $(X,\langle\cdot, \cdot\rangle)$ be a separable Hilbert space endowed with the inner product $\langle\cdot, \cdot\rangle$, which generates the complete norm $\|\cdot\|$, and let $\left\{e_{i}\right\}_{i=1}^{\infty}$ be an orthonormal basis in $X$. In other words, for every natural number $i$, we have $\left\|e_{i}\right\|=1$, and for every pair of natural numbers $j>i$, we have $\left\langle e_{i}, e_{j}\right\rangle=0$.

Let $K$ of the set of all points $x \in X$ such that for every natural numbers $i$,

$$
\left\langle x, e_{2 i-1}\right\rangle \in\left[0, i^{-1}\right]
$$

and

$$
\left\langle x, e_{2 i}\right\rangle \in\left[0, i^{-2}\right] .
$$


Evidently, $K$ is a bounded, closed and convex subset of $X$. It is not difficult to see that the set

$$
\begin{gathered}
K-K=\left\{x \in X:\left|\left\langle x, e_{2 i-1}\right\rangle\right| \leq i^{-1}\right. \\
\left.\left|\left\langle x, e_{2 i}\right\rangle\right| \leq i^{-2} \text { for every natural number } i\right\}
\end{gathered}
$$

is bounded, closed and convex. It is well known that there exists a projection $P: X \rightarrow K-K$ such that for every point $x \in X$, we have

$$
\|P(x)-x\| \leq\|y-x\| \text { for every point } y \in K-K
$$

and such that

$$
\left\|P\left(y_{1}\right)-P\left(y_{2}\right)\right\| \leq\left\|y_{1}-y_{2}\right\| \text { for all } y_{1}, y_{2} \in X .
$$

For every point $x \in K-K$, put

$$
f(x):=\max \left\{\sup \left\{\left|\left\langle x, e_{2 i-1}\right\rangle\right|: i=1,2, \ldots\right\}, \sup \left\{i\left|\left\langle x, e_{2 i}\right\rangle\right|: i=1,2, \ldots\right\}\right\} .
$$

Furthermore, for every point $x \in X \backslash(K-K)$, define

$$
f(x):=f(P(x)) .
$$

Evidently, the function $f: X \rightarrow[0, \infty)$ is well defined, $f(0)=0$, and the set $f(K-K)$ is bounded.

Proposition 1. The function $f: X \rightarrow[0, \infty)$ is uniformly continuous.

Proof. In view of (6), it suffices to show that $f$ is uniformly continuous on $K-K$. Let a positive number $\epsilon$ be given. Choose an integer $n_{0} \geq 1$ for which

$$
n_{0}^{-1}<\epsilon / 4
$$

and then fix a positive number

$$
\delta<\epsilon n_{0}^{-1}
$$

Assume that

$$
z_{1}, z_{2} \in K-K \text { and }\left\|z_{1}-z_{2}\right\| \leq \delta
$$

We claim that

$$
\left|f\left(z_{1}\right)-f\left(z_{2}\right)\right| \leq \epsilon .
$$

Indeed, we may suppose without any loss of generality that

$$
f\left(z_{2}\right) \geq f\left(z_{1}\right) .
$$

It follows from (4) and (7) that there is an integer $j \geq 1$ for which

$$
f\left(z_{2}\right)=\max \left\{\left|\left\langle z_{2}, e_{2 j-1}\right\rangle\right|, j\left|\left\langle z_{2}, e_{2 j}\right\rangle\right|\right\} .
$$

There are two cases: $j>n_{0}$ and $j \leq n_{0}$. First, assume that

$$
j>n_{0} .
$$

Then, by (4), (8), (10), (12) and (13), we have

$$
f\left(z_{2}\right) \leq j^{-1}<n_{0}^{-1}<\epsilon / 4
$$


and

$$
f\left(z_{1}\right) \geq 0 \geq f\left(z_{2}\right)-\epsilon
$$

Next, assume that

$$
j \leq n_{0}
$$

It then follows from (9), (10), (12) and (14) that

$$
\begin{gathered}
f\left(z_{1}\right) \geq \max \left\{\left|\left\langle z_{1}, e_{2 j-1}\right\rangle\right|, j\left|\left\langle z_{1}, e_{2 j}\right\rangle\right|\right\} \\
\geq \max \left\{\left|\left\langle z_{2}, e_{2 j-1}\right\rangle\right|, j\left|\left\langle z_{2}, e_{2 j}\right\rangle\right|\right\}-j\left\|z_{2}-z_{1}\right\| \\
\geq f\left(z_{2}\right)-n_{0}\left\|z_{1}-z_{2}\right\| \geq f\left(z_{2}\right)-n_{0} \delta \geq f\left(z_{2}\right)-\epsilon .
\end{gathered}
$$

Proposition 1 has been established.

Proposition 1 implies property (iii) (see Section 1).

Proposition 2. For every point $x \in K-K$ and every positive number $\lambda<1$, we have

$$
f(\lambda x) \leq \lambda f(x) .
$$

Proof. Assume that $x \in K-K$ and that $\lambda \in(0,1)$. Clearly, there is an integer $j \geq 1$ such that

$$
f(\lambda x)=\max \left\{\lambda\left|\left\langle x, e_{2 j-1}\right\rangle\right|, \lambda j\left|\left\langle x, e_{2 j}\right\rangle\right|\right\} .
$$

By (7) and (15),

$$
f(\lambda x)=\lambda \max \left\{\left|\left\langle x, e_{2 j-1}\right\rangle\right|, j\left|\left\langle x, e_{2 j}\right\rangle\right|\right\} \leq \lambda f(x) .
$$

Proposition 2 has been proved.

Proposition 2 implies property (ii) (see Section 1).

Proposition 3. Let $\epsilon \in(0,1)$ be given. Then there is a positive number $\delta$ such that for every $x \in K-K$ satisfying $f(x) \leq \delta$, the inequality $\|x\| \leq \epsilon$ is true.

Proof. First fix an integer $n_{0} \geq 1$ for which

$$
\sum_{i=n_{0}}^{\infty} i^{-2}<\epsilon^{2} / 8
$$

and then fix $\delta \in(0,1)$ satisfying

$$
16 \delta n_{0}<\epsilon^{2} .
$$

Let

$$
x \in K-K
$$

satisfy

$$
f(x) \leq \delta .
$$

By (7), (18) and (19), we have

$$
\left|\left\langle x, e_{i}\right\rangle\right| \leq \delta, i=1,2, \ldots
$$

In view of (4), (16), (17) and (20), we also have

$$
\|x\|^{2}=\sum_{i=1}^{\infty}\left|\left\langle x, e_{i}\right\rangle\right|^{2}
$$




$$
\begin{gathered}
\leq \sum_{i=1}^{2 n_{0}}\left|\left\langle x, e_{i}\right\rangle\right|^{2}+\sum_{i=2 n_{0}+1}^{\infty}\left|\left\langle x, e_{i}\right\rangle\right|^{2} \\
\leq 2 n_{0} \delta+2 \sum_{i=2 n_{0}+1}^{\infty} i^{-2}<\epsilon^{2} .
\end{gathered}
$$

Proposition 3 is proved.

Proposition 3 implies property (i) (see Section 1).

Next, we define a mapping $A: K \rightarrow K$. To this end, let $x \in K$ be given. In view of (2) and (3), there is a unique point $A(x) \in X$ such that for every natural number $i$, we have

$$
\left\langle A(x), e_{2 i-1}\right\rangle=i\left\langle x, e_{2 i}\right\rangle
$$

and

$$
\left\langle A(x), e_{2 i}\right\rangle=i^{-1}\left\langle x, e_{2 i-1}\right\rangle .
$$

Clearly, $A(x) \in K$ for every point $x \in K$.

Proposition 4. For every pair of points $x, y \in K$,

$$
f(A(x)-A(y)) \leq f(x-y)
$$

Proof. Let the points $x, y \in K$ be given. By (7), (21) and (22),

$$
\begin{gathered}
f(A(x)-A(y))=\max \left\{\sup \left\{\left|\left\langle A(x)-A(y), e_{2 i-1}\right\rangle\right|: i=1,2, \ldots\right\},\right. \\
\left.\sup \left\{i\left|\left\langle A(x)-A(y), e_{2 i}\right\rangle\right|: i=1,2, \ldots\right\}\right\} \\
=\max \left\{\sup \left\{i\left|\left\langle x-y, e_{2 i}\right\rangle\right|: i=1,2, \ldots\right\}, \sup \left\{\left|\left\langle x-y, e_{2 i-1}\right\rangle\right|: i=1,2, \ldots\right\}\right\} \\
=f(x-y) .
\end{gathered}
$$

Proposition 4 has been proved.

Proposition 5. The mapping $A: K \rightarrow K$ is uniformly continuous.

Proof. Let $\epsilon$ be a positive number. There is an integer $n_{0}>4$ for which

$$
16 \sum_{i=n_{0}}^{\infty} i^{-2}<\epsilon^{2}
$$

Fix $\delta \in(0,1)$ for which

$$
4 n_{0}^{3} \delta<\epsilon^{2}
$$

and assume that

$$
x, y \in K \text { and }\|x-y\| \leq \delta .
$$

By Equations (2), (3), (21)-(24) and the above relation, we now have

$$
\begin{gathered}
\|A(x)-A(y)\|^{2}=\sum_{i=1}^{\infty}\left\langle e_{i}, A(x)-A(y)\right\rangle^{2} \\
=\sum_{i=1}^{\infty}\left\langle e_{2 i-1}, A(x)-A(y)\right\rangle^{2}+\sum_{i=1}^{\infty}\left\langle e_{2 i}, A(x)-A(y)\right\rangle^{2}
\end{gathered}
$$




$$
\begin{gathered}
\leq \sum_{i=1}^{n_{0}}\left\langle e_{2 i-1}, A(x)-A(y)\right\rangle^{2}+\sum_{i=1}^{n_{0}}\left\langle e_{2 i}, A(x)-A(y)\right\rangle^{2}+8 \sum_{i=n_{0}+1}^{\infty} i^{-2} \\
\leq \sum_{i=1}^{n_{0}} i^{2}\left\langle e_{2 i}, x-y\right\rangle^{2}+\sum_{i=1}^{n_{0}}\left\langle e_{2 i-1}, x-y\right\rangle^{2}+\epsilon^{2} / 2 \\
\leq 2 n_{0}^{3}\|x-y\|^{2}+\epsilon^{2} / 2 \leq 2 n_{0}^{3} \delta^{2}+\epsilon^{2} / 2<\epsilon^{2} .
\end{gathered}
$$

Proposition 5 has been established.

It is clear that

$$
A(0)=0,
$$

for each $\lambda \in(0,1]$,

$$
\begin{gathered}
\lambda\left(\sum_{i=1}^{\infty} i^{-1} e_{2 i-1}+\sum_{i=1}^{\infty} i^{-2} e_{2 i}\right) \in K, \\
A\left(\lambda\left(\sum_{i=1}^{\infty} i^{-1} e_{2 i-1}+\sum_{i=1}^{\infty} i^{-2} e_{2 i}\right)\right) \\
=\lambda\left(\sum_{i=1}^{\infty} i^{-1} e_{2 i-1}+\sum_{i=1}^{\infty} i^{-2} e_{2 i}\right),
\end{gathered}
$$

and that for every natural number $i \geq 1$,

$$
i^{-1} e_{2 i-1}, i^{-2} e_{2 i} \in K, A\left(i^{-2} e_{2 i}\right)=i^{-1} e_{2 i-1} .
$$

Hence

$$
\left\|A\left(i^{-2} e_{2 i}\right)\right\|\left(\left\|i^{-2} e_{2 i}\right\|\right)^{-1} \rightarrow \infty \text { as } i \rightarrow \infty .
$$

Therefore the mapping $A: K \rightarrow K$ is not Lipschitz with respect to any norm which is equivalent to the norm induced by the inner product.

Let $x \in K$ be given. In view of (21) and (22), for every natural number $i$,

$$
\left\langle A^{2}(x), e_{2 i-1}\right\rangle=i\left\langle A(x), e_{2 i}\right\rangle=i\left(i^{-1}\left\langle x, e_{2 i-1}\right\rangle\right)=\left\langle x, e_{2 i-1}\right\rangle
$$

and

$$
\left\langle A^{2}(x), e_{2 i}\right\rangle=i^{-1}\left\langle A(x), e_{2 i-1}\right\rangle=i^{-1}\left(i\left\langle x, e_{2 i}\right\rangle\right)=\left\langle x, e_{2 i}\right\rangle .
$$

Thus

$$
A^{2}(x)=x
$$

for every point $x \in K$. Therefore the origin is the unique fixed point of $A$ in $K$, but the iterates of $A$ do not converge to it.

Let $\lambda \in(0,1)$. Define

$$
A_{\lambda}(x):=\lambda A(x), x \in K
$$

Clearly,

$$
A_{\lambda}(K) \subset K
$$

By (21), (22) and (26), for all $x \in K$,

$$
A_{\lambda}(x)=A(\lambda x)
$$

Proposition 4 and (27) imply that

$$
f\left(A_{\lambda}(x)-A_{\lambda}(y)\right)=f(A(\lambda x)-A(\lambda y)) \leq f(\lambda x-\lambda y)=\lambda f(x-y) .
$$


Evidently, (1) holds with $\psi(t)=\lambda$ for every nonnegative number $t$. Theorem 1 implies that the origin is the unique fixed point of $A_{\lambda}: K \rightarrow K$ and that all the iterates of $A_{\lambda}$ converge to it, uniformly on $K$.

It follows from (25) and (26) that for every natural number $i$, we have

$$
A_{\lambda}\left(i^{-2} e_{2 i}\right)=\lambda A\left(i^{-2} e_{2 i}\right)=\lambda i^{-1} e_{2 i-1}
$$

and

$$
\left\|A_{\lambda}\left(i^{-2} e_{2 i}\right)\right\|\left(\left\|i^{-2} e_{2 i}\right\|\right)^{-1}=\lambda i^{-1} /\left(i^{-2}\right) \rightarrow \infty \text { as } i \rightarrow \infty \text {. }
$$

Therefore, the mapping $A_{\lambda}: K \rightarrow K$ is not Lipschitz with respect to any norm which is equivalent to the norm induced by the inner product.

Author Contributions: Writing—original draft, S.R. and A.J.Z. All authors have read and agreed to the published version of the manuscript.

Funding: Simeon Reich was supported by the Israel Science Foundation (Grant No. 820/17), by the Fund for the Promotion of Research at the Technion and by the Technion General Research Fund.

Acknowledgments: Both authors are grateful to two anonymous referees for the close reading of the manuscript, and for their useful comments and helpful suggestions.

Conflicts of Interest: The authors declare no conflict of interest.

\section{References}

1. Banach, S. Sur les opérations dans les ensembles abstraits et leur application aux équations intégrales. Fund. Math. 1922, 3, 133-181. [CrossRef]

2. Betiuk-Pilarska, A.; Benavides, T.D. Fixed points for nonexpansive mappings and generalized nonexpansive mappings on Banach lattices. Pure Appl. Funct. Anal. 2016, 1, 343-359.

3. Butnariu, D.; Reich, S.; Zaslavski, A.J. Convergence to fixed points of inexact orbits of Bregman-monotone and of nonexpansive operators in Banach spaces. In Fixed Point Theory and Its Applications; Yokohama Publishers: Yokohama, Japan, 2006; pp. 11-32.

4. de Blasi, F.S.; Myjak, J.; Reich, S.; Zaslavski, A.J. Generic existence and approximation of fixed points for nonexpansive set-valued maps. Set-Valued Var. Anal. 2009, 17, 97-112. [CrossRef]

5. Goebel, K.; Kirk, W.A. Topics in Metric Fixed Point Theory; Cambridge University Press: Cambridge, UK, 1990.

6. Goebel, K.; Reich, S. Uniform Convexity, Hyperbolic Geometry, and Nonexpansive Mappings; Marcel Dekker: New York, NY, USA; Basel, Switzerland, 1984.

7. Jachymski, J. Extensions of the Dugundji-Granas and Nadler's theorems on the continuity of fixed points. Pure Appl. Funct. Anal. 2017, 2, 657-666.

8. Kirk, W.A. Contraction mappings and extensions. In Handbook of Metric Fixed Point Theory; Kluwer: Dordrecht, The Netherlands, 2001; pp. 1-34.

9. Kubota, R.; Takahashi, W.; Takeuchi, Y. Extensions of Browder's demiclosedness principle and Reich's lemma and their applications. Pure Appl. Funct. Anal. 2016, 1, 63-84.

10. Reich, S.; Zaslavski, A.J. Well-posedness of fixed point problems. Far East J. Math. Sci. Spec. Vol. Funct. Anal. Appl. 2001, 46, 393-401. [CrossRef]

11. Pustylnyk, E.; Reich, S.; Zaslavski, A.J. Convergence to compact sets of inexact orbits of nonexpansive mappings in Banach and metric spaces. Fixed Point Theory Appl. 2008, 2008, 528614. [CrossRef]

12. Reich, S.; Zaslavski, A.J. Generic aspects of metric fixed point theory. In Handbook of Metric Fixed Point Theory; Kluwer: Dordrecht, The Netherlands, 2001; pp. 557-575.

13. Reich, S.; Zaslavski, A.J. Convergence to attractors under perturbations. Commun. Math. Anal. 2011, 10, 57-63.

14. Reich, S.; Zaslavski, A.J. Genericity in nonlinear analysis. In Developments in Mathematics; Springer: New York, NY, USA, 2014; Volume 34.

15. Zaslavski, A.J. Approximate solutions of common fixed point problems. In Springer Optimization and Its Applications; Springer: Cham, Switzerland, 2016.

16. Zaslavski, A.J. Algorithms for solving common fixed point problems. In Springer Optimization and Its Applications; Springer: Cham, Switzerland, 2018. 
17. Censor, Y.; Zaknoon, M. Algorithms and convergence results of projection methods for inconsistent feasibility problems: A review. Pure Appl. Funct. Anal. 2018, 3, 565-586.

18. Gibali, A. A new split inverse problem and an application to least intensity feasible solutions. Pure Appl. Funct. Anal. 2017, 2, 243-258.

19. Gibali, A.; Reich, S.; Zalas, R. Outer approximation methods for solving variational inequalities in Hilbert space. Optimization 2017, 66, 417-437. [CrossRef]

20. Takahashi, W. The split common fixed point problem and the shrinking projection method for new nonlinear mappings in two Banach spaces. Pure Appl. Funct. Anal. 2017, 2, 685-699.

21. Takahashi, W. A general iterative method for split common fixed point problems in Hilbert spaces and applications. Pure Appl. Funct. Anal. 2018, 3, 349-369.

22. Gabour, M.; Reich, S.; Zaslavski, A.J. A generic fixed point theorem. Indian J. Math. 2014, 56, 25-32.

23. Reich, S.; Zaslavski, A.J. Contractivity and genericity results for a class of nonlinear mappings. J. Nonlinear Convex Anal. 2015, 16, 1113-1122.

24. de Blasi, F.S.; Myjak, J. Sur la convergence des approximations successives pour les contractions non linéaires dans un espace de Banach. C. R. Acad. Sci. Paris 1976, 283, 185-187.

25. Reich, S.; Zaslavski, A.J. Well-posedness of generalized best approximation problems. Nonlinear Funct. Anal. Appl. 2002, 7, 115-128.

26. Reich, S.; Zaslavski, A.J. Porous sets and generalized best approximation problems. Nonlinear Anal. Forum 2004, 9, 135-152.

27. Reich, S.; Zaslavski, A.J. Existence of a unique fixed point for nonlinear contractive mappings. Mathematics 2020, 8, 55. [CrossRef]

28. Rakotch, E. A note on contractive mappings. Proc. Am. Math. Soc. 1962, 13, 459-465. [CrossRef]

(C) 2020 by the authors. Licensee MDPI, Basel, Switzerland. This article is an open access article distributed under the terms and conditions of the Creative Commons Attribution (CC BY) license (http:/ / creativecommons.org/licenses/by/4.0/). 